\title{
Nebulized antibiotics for ventilator-associated pneumonia: misleading analysis and interpretation of the data
}

Wan-Jie Gu

See related research by Zampieri et al., http://ccforum.com/content/19/1/150

I read with interest the recent systematic review of nebulized antibiotics for ventilator-associated pneumonia [1]. I congratulate and applaud Zampieri and colleagues' important work, but several important issues should be noted.

First, there is one important methodological issue with the review. The data from randomized controlled trials and observational studies were inappropriately pooled together, which goes against the precept of pooling studies with similar design. In fact, the pooling of data from randomized controlled trials and observational studies is only recommended for the assessment of harm/adverse effects. Thus, results from randomized controlled trials and results from observational studies should be separately pooled.

Second, two of the included observational studies were case-control studies [2,3], and therefore using the relative risk as the summary statistic is improper. Instead, the odds ratio should be used as the summary statistic to pool data from observational studies.

Third, the authors used the standardized mean difference as the summary statistic for the continuous variables. As we know, the standardized mean difference is unitless because it is a relative, rather than an absolute, measure of effect. Instead, the authors should have used the mean difference.

Last, Kalin and colleagues' study should be excluded from the review. This study does not state why patients received inhaled colistin and how many patients received a high, normal or low dose in the inhaled plus intravenous colistin group and the intravenous colistin group [4]. Inadequate allocation may thus exist, resulting in a significant potential for selection bias. Inclusion of this study leads to significant heterogeneity, as shown in the forest plot for clinical cure.

\section{Competing interests}

The author declares that he has no competing interests.

\section{Author's contribution}

WJG conceived the study, participated in the design, collected the data, and drafted the manuscript. The author read and approved the final manuscript.

Published online: 15 June 2015

\section{References}

1. Zampieri FG, Nassar AP, Gusmao-Flores D, Taniguchi LU, Torres A, Ranzani OT. Nebulized antibiotics for ventilator-associated pneumonia: a systematic review and meta-analysis. Crit Care. 2015;19:150.

2. Ghannam DE, Rodriguez GH, Raad II, Safdar A. Inhaled aminoglycosides in cancer patients with ventilator-associated Gram-negative bacterial pneumonia: safety and feasibility in the era of escalating drug resistance. Eur J Clin Microbiol Infect Dis. 2009;28:253-9.

3. Kofteridis DP, Alexopoulou C, Valachis A, Maraki S, Dimopoulou D, Georgopoulos D, et al. Aerosolized plus intravenous colistin versus intravenous colistin alone for the treatment of ventilator-associated pneumonia: a matched case-control study. Clin Infect Dis. 2010;51:1238-44.

4. Kalin G, Alp E, Coskun R, Demiraslan H, Gundogan K, Doganay M. Use of high-dose IV and aerosolized colistin for the treatment of multidrug-resistant Acinetobacter baumannii ventilator-associated pneumonia: do we really need this treatment? J Infect Chemother. 2012;18:872-7.

Correspondence: wanjiegu@hotmail.com

Department of Anesthesiology, Affiliated Drum Tower Hospital, Medical College of Nanjing University, 321 Zhong Shan Road, Nanjing 210008, Jiangsu, China 